\title{
A FORMULA FOR THE $r$-COLOURED PARTITION FUNCTION IN TERMS OF THE SUM OF DIVISORS FUNCTION AND ITS INVERSE
}

\author{
SUMIT KUMAR JHA
}

\begin{abstract}
Let $p_{-r}(n)$ denote the $r$-coloured partition function, and $\sigma(n)=\sum_{d \mid n} d$ denote the sum of positive divisors of $n$. The aim of this note is to prove the following

$p_{-r}(n)=\theta(n)+\sum_{k=1}^{n-1} \frac{r^{k+1}}{(k+1) !} \sum_{\alpha_{1}=k}^{n-1} \sum_{\alpha_{2}=k-1}^{\alpha_{1}-1} \cdots \sum_{\alpha_{k}=1}^{\alpha_{k-1}-1} \theta\left(n-\alpha_{1}\right) \theta\left(\alpha_{1}-\alpha_{2}\right) \cdots \theta\left(\alpha_{k-1}-\alpha_{k}\right) \theta\left(\alpha_{k}\right)$

where $\theta(n)=n^{-1} \sigma(n)$, and its inverse

$$
\sigma(n)=n \sum_{r=1}^{n} \frac{(-1)^{r-1}}{r}\left(\begin{array}{l}
n \\
r
\end{array}\right) p_{-r}(n) .
$$
\end{abstract}

\section{MAin RESUlts}

Definition 1. [1] A partition of a positive integer $n$ is a finite weakly decreasing sequence of positive integers $\lambda_{1} \geq \lambda_{2} \geq \cdots \geq \lambda_{r}>0$ such that $\sum_{i=1}^{r} \lambda_{r}=n$. Let $p(n)$ denote the number of partitions of $n$.

A partition is said to be $r$-coloured if each part can occur as $r$ colours. Let $p_{-r}(n)$ denote the number of $r$ coloured partitions of $n$. The generating function for $p_{-r}(n)$ is given by

$$
\sum_{n=0}^{\infty} p_{-r}(n) q^{n}=E(q)^{-r}
$$

where

$$
E(q):=\prod_{j=1}^{\infty}\left(1-q^{j}\right)
$$

where $|q|<1$.

In the following, let $\sigma(n)=\sum_{d \mid n} d$ denote the sum of divisors of a positive integer $n$.

We first prove the following.

2010 Mathematics Subject Classification. 11P81.

Key words and phrases. Partition function; Divisor function; Bell polynomials; r-coloured Partition. 
Theorem 1. For all positive integers $n \geq 2$ we have

$p_{-r}(n)=\theta(n)+\sum_{k=1}^{n-1} \frac{r^{k+1}}{(k+1) !} \sum_{\alpha_{1}=k}^{n-1} \sum_{\alpha_{2}=k-1}^{\alpha_{1}-1} \cdots \sum_{\alpha_{k}=1}^{\alpha_{k-1}-1} \theta\left(n-\alpha_{1}\right) \theta\left(\alpha_{1}-\alpha_{2}\right) \cdots \theta\left(\alpha_{k-1}-\alpha_{k}\right) \theta\left(\alpha_{k}\right)$

where $\theta(n)=n^{-1} \sigma(n)$.

Lemma 1. We have

$$
-\log (E(q))=\sum_{n=1}^{\infty} \theta(n) q^{n}
$$

Proof. It is easy to see that

$$
\begin{aligned}
\log (E(q)) & =\sum_{j=1}^{\infty} \log \left(1-q^{j}\right) \\
& =-\sum_{j=1}^{\infty} \sum_{l=1}^{\infty} \frac{q^{l j}}{l} \\
& =-\sum_{n=1}^{\infty} q^{n}\left(\sum_{d \mid n} \frac{1}{d}\right) .
\end{aligned}
$$

This completes the proof.

Definition 2. For $n$ and $k$ non-negative integers, the partial $(n, k)$ th partial Bell polynomials in the variables $x_{1}, x_{2}, \ldots, x_{n-k+1}$ denoted by $B_{n, k} \equiv \mathrm{B}_{n, k}\left(x_{1}, x_{2}, \ldots, x_{n-k+1}\right)[3, \mathrm{p}$. 206] can be defined by

$$
\mathrm{B}_{n, k}\left(x_{1}, x_{2}, \ldots, x_{n-k+1}\right)=\sum_{\substack{1 \leq i \leq n, \ell_{i} \in \mathbb{N} \\ \sum_{n=1}^{n} i=1 \\ \sum_{i=1} \ell_{i}=k}} \frac{n !}{\prod_{i=1}^{n-k+1} \ell_{i} !} \prod_{i=1}^{n-k+1}\left(\frac{x_{i}}{i !}\right)^{\ell_{i}} .
$$

Cvijovic [2] gives the following formula for calculating these polynomials

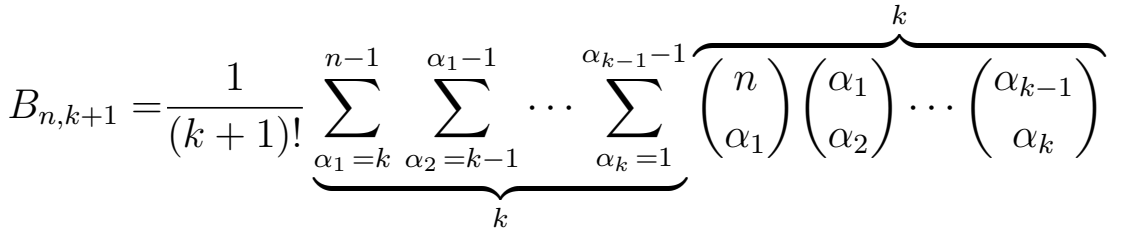

$$
\begin{aligned}
& \cdot x_{n-\alpha_{1}} x_{\alpha_{1}-\alpha_{2}} \cdots x_{\alpha_{k-1}-\alpha_{k}} x_{\alpha_{k}} \quad(n \geq k+1, k=1,2, \ldots)
\end{aligned}
$$

Lemma 2. We have

$$
p_{-r}(n)=\frac{1}{n !} \sum_{k=1}^{n} r^{k} B_{n, k}(1 ! \theta(1), 2 ! \theta(2), \cdots,(n-k+1) ! \theta(n-k+1)),
$$


A FORMULA FOR THE $r$-COLOURED PARTITION FUNCTION IN TERMS OF THE SUM OF DIVISORS FUNCTION where $\theta(n)=n^{-1} \sigma(n)$.

Proof. Let $f(q)=e^{r q}$, and $g(q)=-\log (E(q))$. Using Faà di Bruno's formula [3, p. 134] we have

$$
\frac{d^{n}}{d q^{n}} f(g(q))=\sum_{k=1}^{n} f^{(k)}(g(q)) \cdot B_{n, k}\left(g^{\prime}(q), g^{\prime \prime}(q), \ldots, g^{(n-k+1)}(q)\right) .
$$

Since $f^{(k)}(q)=r^{k} e^{r q}$ and $g(0)=1$, letting $q \rightarrow 0$ in the above equation gives us equation (3).

Combining equations (3) and (2) we can conclude (1).

Now we prove the following.

Theorem 2. We have

$$
\sigma(n)=n \sum_{r=1}^{n} \frac{(-1)^{r-1}}{r}\left(\begin{array}{l}
n \\
r
\end{array}\right) p_{-r}(n)
$$

Lemma 3. We have

$$
\theta(n)=\frac{1}{n !} \sum_{k=1}^{n}(-1)^{k-1}(k-1) ! B_{n, k}(1 ! p(1), 2 ! p(2), \cdots,(n-k+1) ! p(n-k+1))
$$

where $p(n)=p_{-1}(n)$ is the partition function.

Proof. Let $f(q)=\log q$, and $g(q)=1 / E(q)$. Then using Faà di Bruno's formula (4) we have

$$
\frac{d^{n}}{d q^{n}} f(g(q))=\sum_{k=1}^{n} f^{(k)}(g(q)) \cdot B_{n, k}\left(g^{\prime}(q), g^{\prime \prime}(q), \ldots, g^{(n-k+1)}(q)\right) .
$$

Since $f^{(k)}(q)=\frac{(-1)^{k-1}(k-1) !}{q^{k}}$ and $g(0)=1$, letting $q \rightarrow 0$ in the above equation gives us equation (6).

Lemma 4. We have for positive integers $n, k$

$$
B_{n, k}(1 ! p(1), 2 ! p(2), \cdots,(n-k+1) ! p(n-k+1))=\frac{n !}{k !} \sum_{r=1}^{k}(-1)^{k-r}\left(\begin{array}{l}
k \\
r
\end{array}\right) p_{-r}(n)
$$

Proof. We start with the generating function for the partial Bell polynomials as follows

$$
\begin{aligned}
\sum_{n=k}^{\infty} B_{n, k}(1 ! p(1), 2 ! p(2), \cdots,(n-k+1) ! p(n-k+1)) \frac{q^{n}}{n !} & =\frac{1}{k !}\left(\sum_{j=1}^{\infty} p(j) q^{j}\right)^{k} \\
& =\frac{1}{k !}\left(E(q)^{-1}-1\right)^{k} \\
& =\frac{1}{k !} \sum_{r=0}^{k}(-1)^{k-r}\left(\begin{array}{l}
k \\
r
\end{array}\right) E(q)^{-r}
\end{aligned}
$$




$$
=\frac{1}{k !} \sum_{r=0}^{k}(-1)^{k-r}\left(\begin{array}{l}
k \\
r
\end{array}\right) \sum_{n=0}^{\infty} p_{-r}(n) q^{n}
$$

to conclude the equation (7).

Proof of Theorem 2. Combining equations (6) and (7) we have

$$
\begin{aligned}
\sum_{d \mid n} \frac{1}{d} & =\sum_{k=1}^{n} \frac{1}{k} \sum_{r=1}^{k}(-1)^{r-1}\left(\begin{array}{l}
k \\
r
\end{array}\right) p_{-r}(n) \\
& =\sum_{r=1}^{n}(-1)^{r-1} p_{-r}(n) \sum_{k=r}^{n} \frac{1}{k}\left(\begin{array}{l}
k \\
r
\end{array}\right) \\
& =\sum_{r=1}^{n} \frac{(-1)^{r-1}}{r}\left(\begin{array}{l}
n \\
r
\end{array}\right) p_{-r}(n) .
\end{aligned}
$$

Now we can conclude our main result equation (5) after the fact that $\sum_{d \mid n} \frac{n}{d}=\sum_{j \mid n} j$.

\section{REFERENCES}

[1] S. Chern, S. Fu \& D. Tang, Some inequalities for k-colored partition functions, Ramanujan J 46 , $713-725(2018)$

[2] D. Cvijović, New identities for the partial Bell polynomials, Applied mathematics letters, 24 (2011), 1544-1547.

[3] L. Comtet, Advanced Combinatorics: The Art of Finite and Infinite Expansions, D. Reidel Publishing Co., Dordrecht, 1974.

International Institute of Information TeChnology, Hyderabad, India

Email address: kumarjha.sumit@research.iiit.ac.in 